\title{
Hierarchical Bayesian Approach to Boundary Value Problems with Stochastic Boundary Conditions
}

\author{
Christopher K. Wikle* \\ Department of Statistics, University of Missouri \\ L. Mark Berliner \\ Department of Statistics, Ohio State University \\ Ralph F. Milliff \\ Colorado Research Associates, \\ a Division of NorthWest Research Associates, Inc.
}

Submitted to: Monthly Weather Review

August 2002

${ }^{*}$ Corresponding author: Christopher K. Wikle, Department of Statistics, University of Missouri, 222 Math Science Building, Columbia, MO 65211; wikle@stat.missouri.edu 


\begin{abstract}
Boundary value problems are ubiquitous in the atmospheric and ocean sciences. Typical settings include bounded, partially bounded, global and limited area domains, discretized for applications of numerical models of the relevant fluid equations. Often, limited area models are constructed to interpret intensive datasets collected over a specific region, from a variety of observational platforms. These data are noisy and they typically do not span the domain of interest uniformly in space and time. Traditional numerical procedures cannot easily account for these uncertainties. A hierarchical Bayesian modeling framework is developed for solving boundary value problems in such settings. By allowing the boundary process to be stochastic, and conditioning the interior process on this boundary, one can account for the uncertainties in the boundary process in a reasonable fashion. In the presence of data and all its uncertainties, this idea can be related through Bayes' Theorem to produce distributions of the interior process given the observational data. The method is illustrated with an example of obtaining atmospheric streamfunction fields in the Labrador Sea region, given scatterometer-derived observations of the surface wind field.
\end{abstract}




\section{Introduction}

Spatial models, both deterministic and stochastic, are used in atmosphere and ocean sciences in applications such as state estimation, diagnostic analyses, syntheses of field observations from intensive field programs, data assimilation, and process modeling. Considering that data typically exhibit measurement error and bias, and that physical assumptions are often approximate, there has been growing interest in both the statistics and atmospheric/ocean science communities regarding stochastic models that utilize physical information.

The Bayesian paradigm is useful for combining different sources of information

(e.g., physics and data) and accounting for uncertainty. However, for complicated geophysical processes, it is often difficult to specify realistic models and implement them from the Bayesian perspective. Recently, it has been shown that hierarchical approaches to such models provide an ideal framework in which to include physically based prior information for certain geophysical processes (e.g., Wikle et al. 1998; Royle et al. 1999; Wikle et al. 2001). However, in such studies the treatment of boundary or edge effects is problematic and is often somewhat ad hoc.

This paper describes methods for the incorporation of realistic boundary models in a hierarchical Bayesian framework. The methodology is illustrated with the problem of generating distributions of atmospheric streamfunction fields over limited spatial domains, given incomplete satellite observations of surface wind over the Labrador Sea. While atmospheric streamfunction at the surface might not be a quantity that is often employed in traditional analysis methods relevant to air-sea dynamics, we use it here for two reasons. First, the surface streamfunction is a scalar field that relates directly to the surface vector wind observations from scatterometer. Second, in a later paper, we extend the Bayesian hierarchical model methods introduced here to demonstrate a coupled air-sea model wherein surface streamfunction plays a significant role (Berliner et al. 2002). 
The next section describes the hierarchical Bayesian modeling approach in general, and then relative to the boundary value problem. A simple illustration is included to show how the methodology can be applied. Section 3 contains the application of this methodology to the problem of finding the distribution of atmospheric streamfunction fields near the surface, given satellite observations of surface winds over a limited area in the Labrador Sea. Finally, Section 4 contains a discussion.

\section{Methodology}

The Bayesian statistical paradigm is based in probability theory (e.g., Berger 1985; Bernardo and Smith 1994). Assume we are interested in some process $\mathbf{y}$ and we have observational data for this process, denoted by z. Furthermore, there are parameters associated with our physical-statistical representation of the $\mathbf{y}$ process, as well as the statistical model for the observations. The collection of these parameters is denoted by $\boldsymbol{\theta}$. A Bayesian hierarchical analysis develops a joint probability model for all these variables as the product of a sequence of distributions; formally,

$$
[\mathbf{z}, \mathbf{y}, \boldsymbol{\theta}]=[\mathbf{z} \mid \mathbf{y}, \boldsymbol{\theta}][\mathbf{y} \mid \boldsymbol{\theta}][\boldsymbol{\theta}]
$$

where the brackets [ ] denote probability distribution and vertical bars | identify conditional dependencies for a given process upon other processes and/or parameters. For example, $[\mathbf{z} \mid \mathbf{y}, \boldsymbol{\theta}]$ denotes the distribution of the data $\mathbf{z}$ conditional on the process $\mathbf{y}$ and parameters $\boldsymbol{\theta}$. Updating or learning about the unknown quantities

of interest relies on the probability relationship (Bayes' Theorem):

$$
[\mathbf{y}, \boldsymbol{\theta} \mid \mathbf{z}] \propto[\mathbf{z} \mid \mathbf{y}, \boldsymbol{\theta}][\mathbf{y} \mid \boldsymbol{\theta}][\boldsymbol{\theta}]
$$


We can make use of physical relationships to aid in the specifications of the "prior distributions" $[\mathbf{y} \mid \boldsymbol{\theta}]$ and $[\boldsymbol{\theta}]$. Our interest is with the left-hand side (LHS) of (2), the so-called "posterior distribution", which is the focus of Bayesian analysis. This distribution of the process and parameters given the data updates the prior formulations in light of the observed data. For instance, if the process consists of winds $\mathbf{u}, \mathbf{v}$, and pressure $\mathbf{P}$, we could exploit the geostrophic relationship which would allow us to write a stochastic model for the wind field given the pressure field, $[\mathbf{u}, \mathbf{v} \mid \mathbf{P}, \boldsymbol{\theta}]$. Note that this is a stochastic relationship (i.e., a distribution), which quantifies a source of variability with respect to deviations from the gradient relationship (e.g., $u \propto \partial P / \partial y, v \propto \partial P / \partial x$ ). We can model additional uncertainty by specifying distributions for the parameters $\boldsymbol{\theta}$ as well. For example, the geostrophic model suggests a parameter (to be included as an element of the vector $\boldsymbol{\theta}$ ) that is proportional to the inverse product of the density times the Coriolis term. One might specify this as the prior expected value. A variance about this expected value is then prescribed to generate a distribution for this parameter. The net result is that with relatively simple physical and stochastic representations in the sequence of conditional models (e.g., RHS of (1)), we can obtain a posterior distribution that has very complicated spatial structure; one that, through the quantification of uncertainty, can "adapt" to a wide variety of observations and our prior knowledge of the geophysical system (e.g., Royle et al. 1999).

\subsection{Hierarchical Boundary Value Problem}

We are interested in some spatial process $\{\psi(\mathbf{s}): \mathbf{s} \in D\}$, where $\mathbf{s}$ is a spatial location in $D$, a bounded subset of $d$-dimensional Euclidean space. We assume that some physical model gives a good approximation to the behavior of this process:

$$
g(\psi(\mathbf{s}))=Q_{\mathbf{s}}(\{\zeta(\mathbf{r}): \mathbf{r} \in D\})
$$


where $g$ is a known function and $Q$ is some known functional of a spatial process $\{\zeta(\mathbf{r}): \mathbf{r} \in D\}$, that is thought to be related to $\psi(\mathbf{s})$. In our illustrations, $\psi$ represents streamfunction while $\zeta$ includes a variable essentially equivalent to relative vorticity. Not surprisingly, physical arguments relate the two (e.g., Poisson's equation). However, our fundamental motivation is that we believe the relationship (3) to be approximately correct, rather than exact. In general, such lack of exactness might result from physical simplifications and/or discretization. Furthermore, since the domain $D$ is bounded, we require information about the spatial processes at the domain boundary. In certain physical systems, boundary conditions can be critical and often are not known with certainty. The Bayesian strategy for accounting for a variety of uncertainties arising in the modeling, as well as to efficiently incorporate observational data into the analysis, is to model all unknowns as random variables.

In this article, inference is focused on a $\psi$-process defined on a finite lattice in $D$. We partition this lattice into two pieces: the interior, $I$, and the boundary, $B$. This leads to two vectors, denoted by $\boldsymbol{\psi}_{I}$ and $\boldsymbol{\psi}_{B}$, of primary interest. We develop a prior probability model for the gridded $\psi$-process $\left[\boldsymbol{\psi}_{I}, \boldsymbol{\psi}_{B} \mid \boldsymbol{\theta}\right]$. Hierarchical thinking suggests that this model be formed from two components:

$$
\left[\boldsymbol{\psi}_{I}, \boldsymbol{\psi}_{B} \mid \boldsymbol{\theta}\right]=\left[\boldsymbol{\psi}_{I} \mid \boldsymbol{\psi}_{B}, \boldsymbol{\theta}\right]\left[\boldsymbol{\psi}_{B} \mid \boldsymbol{\theta}\right]
$$

where $\boldsymbol{\theta}$ represents other uncertain, but relevant variables such as $\zeta$ in (3), as well as unknown parameters introduced in the modeling. Note that while the notation and role of randomness may be different from those of the traditional treatment of deterministic boundary value problems, there is a common intuition. In particular, the model $\left[\boldsymbol{\psi}_{I} \mid \boldsymbol{\psi}_{B}, \boldsymbol{\theta}\right]$ prescribes the distribution of interior solutions for fixed boundary conditions. 
Using a specified prior distribution for $\boldsymbol{\theta}$, we seek the posterior distribution

$$
\left[\boldsymbol{\psi}_{I}, \boldsymbol{\psi}_{B}, \boldsymbol{\theta} \mid \mathbf{z}\right] \propto\left[\mathbf{z} \mid \boldsymbol{\psi}_{I}, \boldsymbol{\psi}_{B}, \boldsymbol{\theta}\right]\left[\boldsymbol{\psi}_{I}, \boldsymbol{\psi}_{B} \mid \boldsymbol{\theta}\right][\boldsymbol{\theta}]
$$

where $\mathbf{z}$ refers to "data". This relationship serves as the basis for inference.

\section{$2.2 \quad$ A Simple Illustration}

To demonstrate how one can incorporate hierarchical stochastic boundary pro-

cesses, we consider a very simple illustration. Assume that over some one-dimensional spatial domain $D, \psi$ follows approximately a Poisson equation on the interior:

$$
\frac{d^{2} \psi}{d x^{2}} \approx \zeta
$$

Let $D$ be some bounded interval $D \equiv[0, L]$ with non-homogeneous Dirichlet boundary conditions $\psi(0)$ and $\psi(L)$. Although (6) and the boundary conditions can be solved analytically in simple domains (e.g., Haberman 1987), for illustrative purposes we consider first a deterministic numerical solution of this equation. In particular, we discretize the interval $D$, considering equally-spaced locations $\left\{x_{0}=0, x_{1}, \ldots, x_{n}, x_{n+1}=L\right\}$ and use the finite difference approximation,

$$
\frac{d^{2} \psi}{d x^{2}} \approx \frac{\psi(x+h)-2 \psi(x)+\psi(x-h)}{h^{2}},
$$

where $h=x_{i+1}-x_{i}$. Setting $\psi_{i} \equiv \psi\left(x_{i}\right)$ and $\zeta_{i} \equiv \zeta\left(x_{i}\right)$ and applying (7), finite difference approximations to (6) can be written as

$$
\frac{\psi_{i+1}-2 \psi_{i}+\psi_{i-1}}{h^{2}}=\zeta_{i}, \quad i=1, \ldots, n
$$


Given boundary conditions $\psi_{0}=\psi(0), \psi_{n+1}=\psi(L)$ and $\left\{\zeta_{i}: i=1, \ldots, n\right\}$, a solution to the equations (8) is readily obtained. However, such a solution is subject to uncertainty due to the discretization of the continuous spatial processes. That is, the equations $\psi_{i}=\psi\left(x_{i}\right)$ are really only approximations. Indeed, such discretization impacts are but one of several sources of uncertainty. Others include: (i) our lack of absolute certainty about the use of Poisson's equation for the $\psi$ process; (ii) computational, roundoff errors; and (iii) uncertainty in boundary conditions. In response to such issues, we model the true values of the processes of interest as random variables. We then view basic equations such as the Poisson equation and its approximations (e.g., (8)) as providing information about the probability distributions of the true values. Before proceeding, we point out a potentially confusing, though standard, abuse of notation. Since gridding is so common, we typically maintain notation such as $\psi_{i}=\psi\left(x_{i}\right)$, where now $\psi_{i}$ simply reflects a convenient notation for the gridded, true values of the process, rather than the computed numerical solutions to an approximation of the system.

Pursuing the Poisson-equation example, our attention is now directed to the development of the joint probability distribution of $\left\{\psi_{0}, \psi_{1}, \ldots, \psi_{n}, \psi_{n+1}\right\}$. Further, $\boldsymbol{\zeta}$ is also modeled as a random variable. Let the $\psi$ process at interior and boundary locations be denoted $\boldsymbol{\psi}_{I} \equiv\left(\psi_{1}, \ldots, \psi_{n}\right)^{\prime}$ and $\boldsymbol{\psi}_{B} \equiv\left(\psi_{0}, \psi_{n+1}\right)^{\prime}$, respectively, where I denotes the transpose operation. We develop a probability model (e.g., for the second term on RHS of (5)):

$$
\left[\boldsymbol{\psi}_{I}, \boldsymbol{\psi}_{B} \mid \boldsymbol{\zeta}\right]=\left[\boldsymbol{\psi}_{I} \mid \boldsymbol{\psi}_{B}, \boldsymbol{\zeta}\right]\left[\boldsymbol{\psi}_{B} \mid \boldsymbol{\zeta}\right]
$$

The first distribution on the RHS of (9) can be specified to reflect the physical prior information. We can rewrite (8), moving the boundary points to the RHS:

$$
\left(\psi_{2}-2 \psi_{1}\right) / h^{2} \approx \zeta_{1}-\psi_{0} / h^{2}
$$




$$
\begin{aligned}
\left(\psi_{3}-2 \psi_{2}+\psi_{1}\right) / h^{2} & \approx \zeta_{2} \\
\vdots & \vdots \\
\left(\psi_{n}-2 \psi_{n-1}+\psi_{n-2}\right) / h^{2} & \approx \zeta_{n-1} \\
\left(-2 \psi_{n}+\psi_{n-1}\right) / h^{2} & \approx \zeta_{n}-\psi_{n+1} / h^{2}
\end{aligned}
$$

or, in matrix notation

$$
\mathrm{G} \psi_{I} \approx \zeta+\mathrm{G}_{B} \boldsymbol{\psi}_{B}
$$

where

$$
\mathbf{G} \equiv \frac{1}{h^{2}}\left(\begin{array}{cccccc}
-2 & 1 & 0 & \ldots & \\
1 & -2 & 1 & 0 & \ldots & \\
0 & 1 & -2 & 1 & 0 & \\
& & & \ddots & & \\
& \ldots & 0 & 1 & -2 & 1 \\
& & \ldots & 0 & 1 & -2
\end{array}\right), \mathbf{G}_{B} \equiv \frac{1}{h^{2}}\left(\begin{array}{cc}
-1 & 0 \\
0 & 0 \\
\vdots & \vdots \\
0 & 0 \\
0 & -1
\end{array}\right)
$$

Thus, it is reasonable that $\left[\boldsymbol{\psi}_{I} \mid \boldsymbol{\psi}_{B}, \boldsymbol{\zeta}\right]$ has mean or expected value given by the solution of (12), $E\left(\boldsymbol{\psi}_{I} \mid \boldsymbol{\psi}_{B}, \boldsymbol{\zeta}\right)=\mathbf{G}^{-1}\left(\boldsymbol{\zeta}+\mathbf{G}_{B} \boldsymbol{\psi}_{B}\right)$. Due to the various sources of uncertainty discussed earlier, we quantify anticipated variability about this mean via specification of a distribution such as

$$
\boldsymbol{\psi}_{I} \mid \boldsymbol{\psi}_{B}, \boldsymbol{\zeta} \sim N\left(\mathbf{G}^{-1}\left(\boldsymbol{\zeta}+\mathbf{G}_{B} \boldsymbol{\psi}_{B}\right), \boldsymbol{\Sigma}_{\psi}\right)
$$

where $\sim$ is read "is distributed as" and $N(\boldsymbol{\mu}, \boldsymbol{\Sigma})$ indicates a multivariate normal (or Gaussian) distribution with mean $\boldsymbol{\mu}$ and covariance matrix $\boldsymbol{\Sigma} .^{1}$ In this case, $\Sigma_{\psi}$ is the covariance matrix of the random component of variability not accounted

\footnotetext{
${ }^{1}$ The statement "the conditional distribution of $\mathbf{X}$ given $\mathbf{Y}$ equal to $\mathbf{y}$ is Gaussian (normal) with mean $\boldsymbol{\mu}$ and covariance $\boldsymbol{\Sigma}$ " can be written in three ways: $\mathbf{X} \mid \mathbf{y} \sim N(\boldsymbol{\mu}, \boldsymbol{\Sigma}) ;[\mathbf{X} \mid \mathbf{y}]$ is $N(\boldsymbol{\mu}, \boldsymbol{\Sigma})$; and $\mathbf{X}=\boldsymbol{\mu}+\mathbf{e}$ where $\mathbf{e} \sim N(\mathbf{0}, \boldsymbol{\Sigma})$.
} 
for by the approximate solution of (12). Finally, to complete the probability model (9), we must specify a distribution reflecting our uncertainty about the boundary conditions, say,

$$
\boldsymbol{\psi}_{B} \mid \boldsymbol{\zeta} \sim N\left(\boldsymbol{\mu}_{B}, \mathbf{B}\right)
$$

where, in general, $\boldsymbol{\mu}_{B}$ and $\mathbf{B}$ can depend on $\boldsymbol{\zeta}$. In some cases we may assume that $\boldsymbol{\mu}_{B}$ and $\mathbf{B}$ are known. In this framework, the fixed boundary, often used for deterministic numerical solutions, corresponds to $\mathbf{B}$ as a matrix of zeros (i.e., no variance). We delay comment on the appropriateness of the normal distributions until the following section.

\section{Hierarchical Stochastic Boundary Model Ap- plication}

Air-sea interaction in the Labrador Sea region has been the focus of recent attention from climate scientists because of its role in the ocean deep convection process (e.g., Lab Sea Group 1998; Renfrew et al. 1999). Pre-conditioning for and eventual triggering of ocean deep convection in the Labrador Sea is associated with the formation and propagation of polar lows; intense, local meso-cyclone systems that are poorly resolved in surface analyses from weather centers (Renfrew and Moore 1999; Pagowski and Moore 2001). Of particular interest are the surface wind fields that modulate air-sea fluxes of momentum, heat, and moisture, driving the ocean deep convection process.

In recent years, satellite-borne scatterometer instruments have been able to provide high-resolution, yet spatially and temporally incomplete, wind observations over the world's oceans. In particular, wind estimates from the NASA Scatterometer (NSCAT) provided high-quality surface wind data during its operational lifetime (15 September 1996 - 29 June 1997). Wind data from NSCAT occurred 
in swaths on either side of the polar-orbiting satellite ground track. The orbital passes occurred at approximately 100 minute intervals, precessing westward. Different portions of the Labrador Sea were covered by successive orbits two times in a 24-hour period: ascending passes near 0000 UTC, and descending passes near 1400 UTC. Within the sub-region of the NSCAT swath, observations are reported at $50 \mathrm{~km}$ spatial resolution. Here we continue to use the NSCAT-1 dataset that was used by Royle et al. $(1999)^{2}$.

Consider the NSCAT data shown in Figure 1 for December 26, 1996. Our problem is to predict (spatially) high-resolution (approximately $1 / 2$ degree in latitude and longitude) surface streamfunction fields in the Labrador Sea region given spatially-incomplete surface wind data from NSCAT observations. That is, we seek to predict the distribution of the geophysical process (atmospheric streamfunction) at specified spatial locations, given noisy data (wind) over portions of a limited-area domain of interest.

\subsection{Hierarchical Modeling of Streamfunction}

The Poisson equation arises in many applications in atmospheric science (e.g., Holton 1992, p. 386, 448). For example, to calculate streamfunction from winds, one might first calculate vorticity from the wind field and then solve the Poisson equation numerically, given appropriate boundary conditions. Our problem is complicated by the need to specify boundary values for $\psi$ on the edges of a limitedarea domain given incomplete non-uniform observations of wind, and measurement error in the observed winds. A natural solution to these problems would be to interpolate the winds onto a regular grid and specify an arbitrary boundary condition. Although such a procedure is plausible, it does not account for the random errors

\footnotetext{
${ }^{2}$ These data have been superseded by a $25 \mathrm{~km}$ resolution product as reported by the NASA Scatterometer Project (1998), but the increased spatial resolution is not important to the topic of this paper.
} 
from the various sources of uncertainty in the observations, the discretization, and the interpolation procedure. That is, with ad hoc procedures, one cannot characterize the errors associated with the estimate of the streamfunction. Through a hierarchical boundary-value specification, we can account for these errors and obtain realistic spatial prediction errors for the streamfunction field. Furthermore, we can obtain realizations from the distribution of streamfunction, given the satellite observations. Of course, the prediction errors and realizations depend on the prior specification. It is important to recognize, however, that one is effectively specifying a prior when "solving" the traditional boundary value problem with fixed boundaries. The Bayesian approach simply allows one to account for the uncertainty that one might have about the boundary specification.

\subsubsection{Basic Hierarchical Model}

We develop distributions for $\boldsymbol{\psi}_{I}$ and $\boldsymbol{\psi}_{B}$ (interior and boundary streamfunction, respectively) given the data $\mathbf{U}, \mathbf{V}$. Specifically, $\boldsymbol{\psi}_{I}$ is an $n_{I} \times 1$ vector of streamfunction values at $n_{I}$ spatial locations within the prediction grid of interest; $\boldsymbol{\psi}_{B}$ is an $n_{B} \times 1$ vector of streamfunction values at the $n_{B}$ boundary locations. The data vectors $\mathbf{U}, \mathbf{V}$ are both of dimension $m \times 1$ where the $m$ locations correspond to the NSCAT observation locations as shown in Figure 1.

Following steps like those of Section 2.2, except that the domain of interest is two- rather than one-dimensional, and making explicit reference to the wind data as well as relative vorticity computed from a wind field, we are led to a hierarchical model with the following component distributions:

$$
\begin{gathered}
\mathbf{U}, \mathbf{V} \mid \mathbf{u}, \mathbf{v} \sim N\left(\mathbf{K}\left[\mathbf{u}^{\prime} \mathbf{v}^{\prime}\right]^{\prime}, \boldsymbol{\Sigma}_{\epsilon}\right) \\
\boldsymbol{\psi}_{I} \mid \boldsymbol{\psi}_{B}, \mathbf{u}, \mathbf{v} \sim N\left(\mathbf{G}^{-1}\left(\mathbf{D}_{x} \mathbf{v}-\mathbf{D}_{y} \mathbf{u}+\mathbf{G}_{B} \boldsymbol{\psi}_{B}\right), \boldsymbol{\Sigma}_{\psi}\right),
\end{gathered}
$$




$$
\begin{gathered}
\boldsymbol{\psi}_{B} \sim N\left(\boldsymbol{\mu}_{B}, \mathbf{B}\right), \\
\mathbf{u}, \mathbf{v} \sim N\left(\left[\mu_{u} \mathbf{1}^{\prime} \mu_{v} \mathbf{1}^{\prime}\right]^{\prime}, \boldsymbol{\Sigma}_{u v}\right),
\end{gathered}
$$

where $\mathbf{u}, \mathbf{v}$ are $\left(n_{I}+n_{B}\right) \times 1$ vectors denoting the true wind process at the interior and boundary prediction grid locations. In (17) $\mathbf{D}_{x}, \mathbf{D}_{y}$ correspond to the matrix operators for centered first-difference calculations; thus, $\mathbf{D}_{x} \mathbf{v}-\mathbf{D}_{y} \mathbf{u}$ provides a numerical estimate of relative vorticity. The matrices $\mathbf{G}, \mathbf{G}_{B}$ are the two-dimensional analogs to (13), and $\boldsymbol{\Sigma}_{\psi}$ is the conditional covariance of the streamfunction interior given the streamfunction boundary and the winds. The data model (16) is based on the assumption that all observations within a gridbox centered at a prediction location are assumed to be noisy observations of the true process at that prediction location. This is represented using (i) a $2 m \times 2\left(n_{I}+n_{B}\right)$ incidence matrix (a sparse matrix of ones and zeros) $\mathbf{K}$, and (ii) a measurement error covariance matrix, $\boldsymbol{\Sigma}_{\epsilon}$. Note that more complicated expressions relating observations to the process are possible, leading to more complicated forms for K (e.g., Wikle et al. 1998; Wikle et al. 2001). The distribution (18) requires specification of parameters $\boldsymbol{\mu}_{B}$ and $\mathbf{B}$, the prior mean and covariance matrix, respectively, for the streamfunction boundary process. This specification relies on the simplifying assumption that the boundary process is not directly related to the wind process. Although unrealistic in the present example, this assumption makes possible the analytical derivation of the posterior distribution. Thus, although the method can easily accommodate the more general boundary assumption, we have made the simplifying assumption to facilitate illustration of the approach. Finally, in (19) $\mu_{u}, \mu_{v}$ are the wind component prior means, $\boldsymbol{\Sigma}_{u v}$ is the wind process prior covariance matrix, and $\mathbf{1}$ is a vector of ones. Additional discussion of these distributional parameters is given below.

Our choices of Gaussian distributions for each of the random variables of in- 
terest merit discussion. In the case of the data model (16), we emphasize that it is the measurement errors conditioned on the true wind process that are modeled as Gaussians. This assumption is at least partially justified in the case of NSCAT data by Freilich and Dunbar (1999). The Gaussian assumption for the conditional model on $\boldsymbol{\psi}_{I}$ may be reasonable since it is the deviation from the Poisson relation that is assumed to be Gaussian, not the $\psi_{I}$ process itself. The bivariate Gaussian spatial model on the true wind process (19) is consistent with traditional spatial analysis models (e.g., Daley 1991), although in certain situations one may have to choose the covariance matrix $\boldsymbol{\Sigma}_{u v}$ carefully. Perhaps the least justifiable assumption in the model is the assumption that the boundary process follows a Gaussian spatial model. However, we do not believe such an assumption is unreasonable. Finally, we note that the hierarchical methodology described here is still viable with non-Gaussian distributions, but the Gaussian assumptions allow for analytically tractable calculations of the posterior distribution.

\subsubsection{Bayesian Analyses}

Direct application of Bayes' Theorem provides the posterior distribution

$$
\left[\boldsymbol{\psi}_{I}, \boldsymbol{\psi}_{B}, \mathbf{u}, \mathbf{v} \mid \mathbf{U}, \mathbf{V}\right] \propto[\mathbf{U}, \mathbf{V} \mid \mathbf{u}, \mathbf{v}][\mathbf{u}, \mathbf{v}]\left[\boldsymbol{\psi}_{I} \mid \boldsymbol{\psi}_{B}, \mathbf{u}, \mathbf{v}\right]\left[\boldsymbol{\psi}_{B}\right]
$$

While complete details of the probabilistic calculations are not central to this arti-

cle, a clarifying calculation is presented. Note that the constant of proportionality (referred to as a "normalizing constant") in Bayes' Theorem is given by the integral of the RHS with respect to all variables not in the condition of the LHS. That is, to convert the proportionality to an equality, we divide the RHS of (20) by

$$
\int[\mathbf{U}, \mathbf{V} \mid \mathbf{u}, \mathbf{v}][\mathbf{u}, \mathbf{v}]\left[\boldsymbol{\psi}_{I} \mid \boldsymbol{\psi}_{B}, \mathbf{u}, \mathbf{v}\right]\left[\boldsymbol{\psi}_{B}\right] d \boldsymbol{\psi}_{I} d \boldsymbol{\psi}_{B} d \mathbf{u} d \mathbf{v}=\int[\mathbf{U}, \mathbf{V} \mid \mathbf{u}, \mathbf{v}][\mathbf{u}, \mathbf{v}] d \mathbf{u} d \mathbf{v}
$$


The form of the RHS of (21) is special for this model. The result follows from the previously mentioned simplifying assumption that $\boldsymbol{\psi}_{B}$ is independent of the winds and hence, $\left[\boldsymbol{\psi}_{B}\right]$ is unaffected by the observational data.

Noting that the posterior of the wind process is written

$$
[\mathbf{u}, \mathbf{v} \mid \mathbf{U}, \mathbf{V}]=\frac{[\mathbf{U}, \mathbf{V} \mid \mathbf{u}, \mathbf{v}][\mathbf{u}, \mathbf{v}]}{\int[\mathbf{U}, \mathbf{V} \mid \mathbf{u}, \mathbf{v}][\mathbf{u}, \mathbf{v}] d \mathbf{u} d \mathbf{v}}
$$

we use (21) and (22) to rewrite the posterior distribution (20) as the equality:

$$
\left[\boldsymbol{\psi}_{I}, \boldsymbol{\psi}_{B}, \mathbf{u}, \mathbf{v} \mid \mathbf{U}, \mathbf{V}\right]=[\mathbf{u}, \mathbf{v} \mid \mathbf{U}, \mathbf{V}]\left[\boldsymbol{\psi}_{I} \mid \boldsymbol{\psi}_{B}, \mathbf{u}, \mathbf{v}\right]\left[\boldsymbol{\psi}_{B}\right]
$$

To obtain the posterior distribution of the interior and boundary streamfunction conditional on the wind data, we integrate out the wind process:

$$
\left[\boldsymbol{\psi}_{I}, \boldsymbol{\psi}_{B} \mid \mathbf{U}, \mathbf{V}\right]=\left[\boldsymbol{\psi}_{B}\right] \int\left[\boldsymbol{\psi}_{I} \mid \boldsymbol{\psi}_{B}, \mathbf{u}, \mathbf{v}\right][\mathbf{u}, \mathbf{v} \mid \mathbf{U}, \mathbf{V}] d \mathbf{u} d \mathbf{v}
$$

More often, we are interested in the posterior of the interior process given the data, $\left[\boldsymbol{\psi}_{I} \mid \mathbf{U}, \mathbf{V}\right]$, in which case $\boldsymbol{\psi}_{B}$ is integrated out of (24). In general, such integrations are intractable and must be evaluated with Monte Carlo methods. However, in this example, the choice of distributions (16)-(18) allow analytic determination of this posterior as follows.

Step 1. The posterior of the wind process given the satellite observations $[\mathbf{u}, \mathbf{v} \mid \mathbf{U}, \mathbf{V}]$ can be shown ${ }^{3}$ to be a multivariate normal distribution $N\left(\boldsymbol{\mu}_{u v \mid U V}, \boldsymbol{\Sigma}_{u v \mid U V}\right)$, where

$$
\boldsymbol{\mu}_{u v \mid U V}=\boldsymbol{\Sigma}_{u v \mid U V}\left(\mathbf{K}^{\prime} \boldsymbol{\Sigma}_{\epsilon}^{-1}\left[\mathbf{U}^{\prime} \mathbf{V}^{\prime}\right]^{\prime}+\boldsymbol{\Sigma}_{u v}^{-1}\left[\mu_{u} \mathbf{1}^{\prime} \mu_{v} \mathbf{1}^{\prime}\right]^{\prime}\right),
$$

\footnotetext{
${ }^{3}$ The calculation is accomplished by combining the exponents of the relevant Gaussian density functions and completing the square. In general, if $z \mid y \sim N\left(A y, \Sigma_{z \mid y}\right)$ and $y \sim N\left(\mu_{y}, \Sigma_{y}\right)$ then completing the square gives the posterior distribution $y \mid z \sim N\left(\Sigma_{y \mid z}\left[A^{\prime} \Sigma_{z \mid y}^{-1} z+\Sigma_{y}^{-1} \mu_{y}\right], \Sigma_{y \mid z}\right)$ where $\Sigma_{y \mid z}=\left(A^{\prime} \Sigma_{z \mid y}^{-1} A+\Sigma_{y}^{-1}\right)^{-1}$ (e.g., Berger, 1985).
} 


$$
\boldsymbol{\Sigma}_{u v \mid U V}=\left(\mathbf{K}^{\prime} \boldsymbol{\Sigma}_{\epsilon}^{-1} \mathbf{K}+\boldsymbol{\Sigma}_{u v}^{-1}\right)^{-1}
$$

Step 2. Facts from probability theory ${ }^{4}$ yield the posterior distribution $\left[\boldsymbol{\psi}_{I} \mid \boldsymbol{\psi}_{B}, \mathbf{U}, \mathbf{V}\right]$, which is a multivariate normal distribution $N\left(\boldsymbol{\mu}_{\psi \mid U V, B}, \boldsymbol{\Sigma}_{\psi \mid U V, B}\right)$ with

$$
\boldsymbol{\mu}_{\psi \mid U V, B}=\mathbf{G}^{-1} \mathbf{D} \boldsymbol{\mu}_{u v \mid U V}+\mathbf{G}^{-1} \mathbf{G}_{B} \boldsymbol{\psi}_{B}
$$

and

$$
\boldsymbol{\Sigma}_{\psi \mid U V, B}=\boldsymbol{\Sigma}_{\psi}+\mathbf{G}^{-1} \mathbf{D} \Sigma_{u v \mid U V} \mathbf{D}^{\prime} \mathbf{G}^{-1}
$$

where $\mathbf{D} \equiv\left[\begin{array}{ll}-\mathbf{D}_{y} & \mathbf{D}_{x}\end{array}\right]$

Step 3. Finally, we may integrate out the boundary, yielding the final posterior on the interior streamfunction: $\left[\boldsymbol{\psi}_{I} \mid \mathbf{U}, \mathbf{V}\right]$. The result is again a multivariate normal distribution $N\left(\boldsymbol{\mu}_{\psi \mid U V}, \boldsymbol{\Sigma}_{\psi \mid U V}\right)$ with

$$
\boldsymbol{\mu}_{\psi \mid U V}=\mathbf{G}^{-1} \mathbf{D} \boldsymbol{\mu}_{u v \mid U V}+\mathbf{G}^{-1} \mathbf{G}_{B} \boldsymbol{\mu}_{B}
$$

and

$$
\Sigma_{\psi \mid U V}=\Sigma_{\psi \mid U V, B}+\mathbf{G}^{-1} \mathbf{G}_{B} \mathbf{B G}_{B}^{\prime} \mathbf{G}^{-1}
$$

\subsubsection{Examples}

The characterization of uncertainty is directly related to the choice of prior specification. Below, we illustrate with three cases the effect of prior assumptions on the posterior mean, standard deviation, and realizations.

We begin with specification of the parameters for the distributions in the model

\footnotetext{
${ }^{4}$ The theoretical "facts" that imply these results are (i) if $y \mid x$ has mean $H x$ and covariance $\Sigma_{y \mid x}$ and $x$ has mean $\mu$ and covariance $\Sigma_{x}$, the unconditional (i.e., integrating out $x$ ) mean and covariance of $y$ are $H \mu$ and $\Sigma_{y \mid x}+H \Sigma_{x} H^{\prime}$, respectively; (ii) if the conditional distribution of $y \mid x$ is normal; $x$ enters that conditional distribution only in the mean and linearly in the mean; and if the marginal distribution of $x$ is normal, then the marginal distribution of $y$ is normal.
} 
hierarchy. For the Labrador Sea problem we let $\boldsymbol{\Sigma}_{\epsilon}=\sigma_{\epsilon}^{2} \mathbf{I}$ where the satellite measurement error variance is $\sigma_{\epsilon}^{2}=1.7 \mathrm{~m}^{2} \mathrm{~s}^{-2}$, based loosely on the study by Freilich and Dunbar (1999). Furthermore, we let $\boldsymbol{\Sigma}_{u v}=\mathbf{S}_{u v} \otimes \mathbf{R}_{u v}$, where $\mathbf{S}_{u v}$ is a $2 \times 2$ covariance matrix between the $u$ and $v$ wind components, $\mathbf{R}_{u v}$ is an $\left(n_{I}+n_{B}\right) \times\left(n_{I}+\right.$ $\left.n_{B}\right)$ spatial correlation matrix, and $\otimes$ represents the Kronecker product operation. For the elements of $\mathbf{S}_{u v}$, we let the covariance between $u$ and $v$ be $12 \mathrm{~m}^{2} \mathrm{~s}^{-2}$ and variance of $u$ and $v$ be $213 \mathrm{~m}^{2} \mathrm{~s}^{-2}$ and $55 \mathrm{~m}^{2} \mathrm{~s}^{-2}$, respectively. These values were based on data analysis of scatterometer data in the Labrador Sea region.

The spatial correlation matrix $\mathbf{R}_{u v}$ was assumed to be isotropic and from the Matérn class (Matérn 1986; Handcock and Wallis 1994):

$$
r(d)=\frac{1}{2^{\theta_{2}-1} \Gamma\left(\theta_{2}\right)}\left(\frac{2 d \sqrt{\theta_{2}}}{\theta_{1}}\right)^{\theta_{2}} \mathcal{K}_{\theta_{2}}\left(\frac{2 d \sqrt{\theta_{2}}}{\theta_{1}}\right)
$$

where $d$ is the distance between spatial locations, $\Gamma$ is the gamma function and $\mathcal{K}_{\theta_{2}}$ is the modified Bessel function of the third kind and order $\theta_{2}$. This class of correlation functions is useful because of the wide range of behaviors that can be modeled and the interpretability of the parameters. Specifically, $\theta_{1}>0$ is a spatial scale parameter related to the range of dependence and $\theta_{2}>0$ is related to the smoothness of the spatial field. For example, if $\theta_{2}=1 / 2$ then the correlation function simply reduces to an exponential model; as $\theta_{2} \rightarrow \infty$ the function approaches a "Gaussian" correlation function. In general, the correlation function is $\left\lceil\theta_{2}-1\right.$ times mean-squared differentiable, where $\lceil$ is the integer ceiling function. In our case, given that we would like the wind fields to be differentiable in principle, we let $\theta_{2}=2.5$. Furthermore, we set $\theta_{1}=.0337 \mathrm{~km}^{-1}$, which corresponds to observed Labrador sea wind component length scales of approximately $270 \mathrm{~km}$ (Milliff et al. 2002). Note that the Matérn class does not allow negative correlations. This is not generally a problem for the small domain of interest in the current application (Milliff et al. 2002). However, with a larger domain it would be more realistic 
to consider a spatial correlation function that allows negative correlations (e.g., Thiebaux 1975). Note that one must be careful in the specification of the spatial covariance function for the wind process. As discussed by Bennett and Budgell (1987), there are regularity conditions for this spatial structure such that the covariance function must be sufficiently smooth (e.g., differentiable) to guarantee that the second moment of the streamfunction distribution exists as the grid spacing goes to zero. This requirement is satisfied with the correlation model chosen above.

Given our expectation that the variance of the conditional interior streamfunction is small with spatial dependence over relatively small length scales, we let $\boldsymbol{\Sigma}_{\psi}=\sigma_{\psi}^{2} \mathbf{R}_{\psi}$, where $\sigma_{\psi}^{2}=10^{10} \mathrm{~m}^{4} \mathrm{~s}^{-2}$ and $\mathbf{R}_{\psi}$ follow a Matérn model with smoothness parameter equal to 2.5 and spatial dependence parameter $.0566 \mathrm{~km}^{-1}$. Again, as discussed in Bennett and Budgell (1987), one must be careful with the specification of this covariance function as grid spacings go to zero. Regularity conditions suggest that there should be non-trivial spatial structure in this field under such conditions.

Finally, we will specify $\boldsymbol{\mu}_{B}$ and let $\mathbf{B}=\sigma_{B}^{2} \mathbf{R}_{B}$, where $\mathbf{R}_{B}$ is based on a Matérn correlation model with spatial smoothness parameter 3.5 and spatial dependence parameter $\theta_{B}=.0471 \mathrm{~km}^{-1}$, reflecting a moderate amount of spatial dependence.

We now investigate the sensitivity of the streamfunction posterior distribution to choice of $\boldsymbol{\mu}_{B}$ and $\sigma_{B}^{2}$. We consider three cases, as outlined in Table 1. In Case I, $\boldsymbol{\mu}_{B}$ is specified to match (subjectively) the suggested domain inflow and outflow based on visual inspection of the wind data plot (Figure 1). The resulting boundary mean is shown in Figure 2e and Figure 3e (note that the posterior mean of $\boldsymbol{\psi}_{B}$ and the prior mean are the same for the model presented here). Since the determination of the appropriate boundary process by such visual inspection of the noisy and incomplete winds is uncertain, we specify the boundary process prior variance as 
$\sigma_{B}^{2}=1.1 \times 10^{12} \mathrm{~m}^{4} \mathrm{~s}^{-2}$. In general, this is meant to represent the situation where we might have a rough idea of the boundary condition (say, from an analysis field), but allow for substantial uncertainty in that knowledge. Figure 2 shows the posterior mean, standard deviation and realizations from the posterior distribution of the streamfunction field in this case. Note that the posterior mean streamfunction field is reasonable, with flow across the boundary (as suggested by the intersection of the contours with the boundary) and a strong cyclonic circulation. However, there is substantial uncertainty in this field, as suggested by the large posterior standard deviations and the realizations. Of course, the realizations are not as smooth as the posterior mean due to the (i) uncertainty in the wind data, (ii) noise amplification in the vorticity calculation, and (iii) uncertainty in the boundary process. Clearly, inference based on the posterior mean field would be quite different than that based on one of the realizations.

For comparison, consider Case II where all parameters are identical to Case I except that we specify a nearly "fixed" boundary (we do this by setting the boundary variance to an extremely small value, e.g. $\sigma_{B}^{2}=1.0 \mathrm{~m}^{4} \mathrm{~s}^{-2}$ ). That is, we are very certain as to the boundary value. Figure 3 shows the results from this case. Compared to Case I, there is much less variability in the realizations since we are certain about the boundary, and thus, the posterior standard deviations of the interior streamfunction field are much smaller. This is reasonable since we are comparatively certain as to the boundary value. These comparisons show that uncertainty in the boundary can make a substantial impact on realizations of the interior process. A key point is that our methodology permits one to quantify such impacts explicitly.

As another test, consider Case III where again $\sigma_{B}^{2}=1.1 \times 10^{12} \mathrm{~m}^{4} \mathrm{~s}^{-2}$, but now we let $\boldsymbol{\mu}_{B}=\mu_{b} \mathbf{1}$, where $\mu_{b}=-3.5 \times 10^{5} \mathrm{~m}^{2} \mathrm{~s}^{-1}$ (the mean of $\boldsymbol{\mu}_{B}$ shown in the previous examples). That is, we specify the prior mean of the boundary to be 
constant in space. This corresponds to a no-flux boundary condition, which we know to be unrealistic in the atmospheric setting. Figure 4 shows the corresponding output. Clearly, the posterior mean streamfunction field does not allow flow across the boundary in this setting, which is unrealistic. However, the realizations do allow flow across the boundary, illustrating the effect of the uncertain boundary condition upon the realization from the posterior distribution. In this case, the realizations convey uncertainty that is not obvious from examination of the posterior mean. That is, a poor specification of the prior mean was compensated by the data, leading to a reasonable posterior distribution on the interior streamfunction. One might not be interested in the posterior mean in this setting, but could consider realizations from the posterior distribution for purposes of inference.

\subsection{Remarks}

We note that in some contexts the boundary values are actually not of direct interest, but are rather viewed as a nuisance necessary only for developing a model for the interior (e.g., limited area modeling in the atmosphere or ocean). In such cases, it is natural given the new methods presented here to suggest developing a model in which the boundary is "integrated out" à priori. That is, standard probability theory applied in (4) implies that

$$
\left[\boldsymbol{\psi}_{I} \mid \boldsymbol{\theta}\right]=\int\left[\boldsymbol{\psi}_{I} \mid \boldsymbol{\psi}_{B}, \boldsymbol{\theta}\right]\left[\boldsymbol{\psi}_{B} \mid \boldsymbol{\theta}\right] d \boldsymbol{\psi}_{B}
$$

Alternatively, we performed this integration conditional on the observed data in deriving (29) and (30). This exemplifies a fundamental feature of hierarchical modeling. Namely, the LHS of (31) describes our uncertainty in the interior formally in terms of two sources: our uncertainty about the interior if we knew the boundary and our uncertainty regarding the boundary. Further, it is important to note that 
hierarchical thinking leads to the LHS of (31) in a conceptually simple fashion. That is, direct specification of $\left[\boldsymbol{\psi}_{I} \mid \boldsymbol{\theta}\right]$ without first developing the RHS is typically very difficult. On the other hand, this circumstance should be contrasted with our model for the wind process (19). In that case, the joint prior distribution on the entire wind field is simultaneously specified, rather than the result of a windon-the-boundary model coupled to a interior-wind model given the boundary, as was the case for streamfunction. The key difference is that our streamfunction model was designed to make significant use of Poisson's equation. Incorporation of that physics requires both the traditional modeler and the hierarchical modeler to deal with the boundary explicitly. Indeed, should we seek to incorporate a richer model for winds, perhaps based on some physical reasoning that dictates structures among gridded winds, we would likely need a two-step, explicit boundary model as well.

A second critical remark regarding the value in explicitly developing $\left[\boldsymbol{\psi}_{B} \mid \boldsymbol{\theta}\right]$ is that the formulas of probability theory direct us to formulas for updating this distribution, and hence the RHS of (31), should available data, say $d_{\psi_{B}}$, be informative about the boundary. Specifically, one simply replaces $\left[\boldsymbol{\psi}_{B} \mid \boldsymbol{\theta}\right]$ by $\left[\boldsymbol{\psi}_{B} \mid \boldsymbol{\theta}, d_{\psi_{B}}\right]$; this is obtained via Bayes' Theorem.

\section{Discussion}

The hierarchical stochastic boundary condition methodology outlined here is more general than the relatively simple Poisson example might suggest. For example, we may include Neumann or mixed boundary conditions as well as time-varying boundaries. For Neumann boundary conditions one specifies the gradient of the process at the boundary. A common example is the boundary condition, $\partial \psi / \partial n=$ $\alpha(s)$, which states that the normal derivative at the boundary location $s$ of the process $\psi$ is equal to some value of the process $\alpha$ at that location. For bounded 
domains, a standard numerical procedure uses the value of the process outside the boundary (i.e., at a so-called image point) in terms of its interior reflection. For example, in the 1-d example considered in Section 2, we consider the interior locations $x_{1}, \ldots, x_{n}$. At the left end, the Neumann condition would be $\psi_{0}=$ $\psi_{2}-\alpha_{1} 2 h$. In our case, this would suggest a slight modification to $\mathbf{G}$ as well as the introduction of the $\alpha$ process on the RHS of (12). In this implementation a specific boundary process $\boldsymbol{\psi}_{B}$ need not be specified, but is effectively replaced by a discretized $\alpha$ process defined on the boundary.

The random boundary process $\boldsymbol{\psi}_{B}$ is a useful construct in open boundary specifications of the kind required in the experiments described here. Open boundary specifications raise difficult issues in purely deterministic modeling contexts. Rienecker and Miller (1991) demonstrated the sensitivity of domain-scale solutions to errors in open boundary specifications in data assimilation experiments in a quasi-geostrophic ocean model for a limited-area domain. About a decade later, Lermusiaux (1999) and Lermusiaux and Robinson (2001) report many sophistications in ocean model and data assimilation components of the open ocean forecast system that descends from the prior work. Still, the open boundary specification requires specific regional calibration, dependent upon adequate in-situ observations, in the set-up stages of the forecast experiments. Issues of ill-posedness in quasi-geostrophic ocean model applications in limited-area domains have been noted since Bennett and Kloeden (1978). Miller (1984) described the problem in light of theoretical work that had gone on in pure and applied mathematics in the late 1970's and early 1980's. Similarly, the partition into unique streamfunction and velocity potential fields for atmospheric flows observed in limited-area domains requires arbitrary constraints to close the problem (e.g., Lynch 1989). It appears from our preliminary experiments that issues of these kinds that arise in deterministic modeling contexts can be circumvented by the stochastic approach 
presented here. However, more fundamental demonstrations await further work.

One can imagine that a stochastic boundary condition could be useful for regional models that are nested within larger models. In that case, one might assume that the time-varying boundary process for the nested model is given as a distribution with prior mean from the larger model, with suitable variability. This too will be explored elsewhere.

Finally, the model considered here assumed that the distributional parameters were known. Extensions are possible to cover the cases where the parameters are random and there are data to inform the boundary. In these cases the posterior distribution is rarely tractable analytically. However, recent advances in the development and use of Markov chain Monte Carlo (MCMC) have enabled Bayesian treatments of many large and complex problems (e.g., Robert and Casella 2000). Rather than a direct computation of the posterior distribution, one computes successive simulations from a Markov chain ${ }^{5}$ constructed in a fashion that permits the assertion that its stationary distribution coincides with the target posterior. Hence, after some transience or burn-in time, realizations of the chain are viewed as simulated, though dependent, cases from the posterior distribution. For meteorological applications of these notions, see Wikle et al. (1998), Royle et al. (1999), Berliner et al. (2000), and Wikle et al. (2001).

\section{Acknowledgment}

We are grateful for research support from the Oceanography Program of the NASA Earth Science Enterprise administered by Dr. Eric Lindstrom. Specifically, this material is based upon work supported by the NASA/Goddard Space Flight Center

\footnotetext{
${ }^{5}$ Informally, a (one-step) Markov chain is a sequence of random variables evolving over time, with the property that conditional on the history of the sequence through time $t$, the probability distribution of the state at time $t+1$ depends only on the state at time $t$ (e.g., Robert and Casella, 2000).
} 
under Awards NAG5-8344 (MU), NAG5-8455 (OSU), and a contract from the Jet Propulsion Laboratory JPL-1860 to CoRA/NWRA. In addition, CKW and LMB acknowledge support from the U.S. EPA's Science to Achieve Results (STAR) program, Assistance Agreement R827257-01-0; RFM acknowledges support from the QSCAT Science Team, OSU-3A09 to CoRA/NWRA.

\section{References}

Bennett, A.F., and P.E. Kloeden, 1978: Boundary conditions for limited area forecasts. Journal of the Atmospheric Sciences, 35, 990-996.

Bennett, A.F. and W.P. Budgell, 1987: Ocean data assimilation and the Kalman filter: Spatial regularity, Journal of Physical Oceanography, 17, 1583-1601.

Berger, J. O., 1985: Statistical Decision Theory and Bayesian Analysis, SpringerVerlag, New York.

Berliner, L. M., 1996: Hierarchical Bayesian time series models. In Maximum Entropy and Bayesian Methods, K. Hanson and R. Silver (Eds.), Kluwer Academic Publishers, 15-22.

Berliner, L.M., C.K. Wikle, and N. Cressie, 2000: Long-lead prediction of Pacific SSTs via Bayesian Dynamic Modeling. Journal of Climate, 13, 3953-3968.

Berliner, L.M., R.F. Milliff, and C.K. Wikle, 2002: Bayesian hierarchical modeling of air-sea interaction, submitted to Journal Geophysical Research, Oceans.

Bernardo, J.M. and A.F.M. Smith, 1994: Bayesian Theory, John Wiley \& Sons, Inc., New York.

Cressie, N.A.C., 1993: Statistics for Spatial Data, Revised Edition, Wiley, New York. 
Daley, R., 1991: Atmospheric Data Analysis, Cambridge University Press, Cambridge, UK.

Freilich, M.H., and R.S. Dunbar, 1999: The accuracy of NSCAT-1 vector winds: comparisons with national buoy center buoys. Journal of Geophysical Research, Oceans, 104, 11231-11246.

Haberman, R., 1987: Elementary Applied Partial Differential Equations, PrenticeHall, New Jersey.

Handcock, M.S. and J.R. Wallis, 1994: An approach to statistical spatial-temporal modeling of meteorological fields. Journal of the American Statistical Association, 89, $368-378$.

Holton, J.R., 1992: An Introduction to Dynamic Meteorology, Third Edition, San Diego, CA: Academic Press.

Lab Sea Group, 1998: The Labrador Sea Deep Convection Experiment, Bull. Amer. Met. Soc., 70, 2033-2058.

Lermusiaux, P.F.J., 1999: Estimation and study of mesoscale variability in the Strait of Sicily. Dynamics of Atmospheres and Oceans, 29, 255-303.

Lermusiaux, P.F.J, and A.R. Robinson, 2001: Features of dominant mesoscale variability, circulation patterns and dynamics in the Strait of Sicily. Deep Sea Research $I$, in press.

Lynch, P., 1989: Partitioning the wind in a limited domain, Monthly Weather Review, 117, 1492-1500.

Matérn, B., 1986: Spatial Variation (2nd ed.), Lecture Notes in Statistics, 36, Berlin: Springer-Verlag.

Miller, R.N., 1984: Forecasting the ocean's weather: Numerical models for appli- 
cation to oceanographic data. Contemporary Mathematics, 28, 337-345.

Milliff, R.F., P.P. Niiler, J. Morzel, A.E. Sybrandy, D. Nychka, and W.G. Large, 2002: Mesoscale correlation length scales from NSCAT and Minimet surface wind retrievals in the Labrador Sea. Journal of Atmospheric and Oceanic Technology, submitted.

NASA Scatterometer Project, 1998: Science Data Product (NSCAT-2) User's Manual: Overview and Geophysical Data Products, version 1.2, Jet Propulsion Laboratory, California Institute of Technology, publ. D-12985, 78 pp.

Pagowski, M. and G.W.K. Moore, 2001: A numerical study of an extreme cold-air outbreak over the Labrador Sea: Sea ice, air-sea interaction, and development of polar lows, Monthly Weather Review, 129, 47-72.

Renfrew, I.A., G.W.K. Moore, T.R. Holt, S.W. Chang and P. Guest, 1999: Mesoscale forecasting during a field program: Meteorological support of the Labrador Sea Deep Convection Experiment, Bull. Amer. Met. Soc., 80, 605620.

Renfrew, I.A., and G.W.K. Moore, 1999: An extreme cold-air outbreak over the Labrador Sea: Roll vortices and air-sea interaction, Mon. Wea. Rev., 127, 2379-2394.

Rienecker, M.M., and R.N. Miller, 1991: Ocean data assimilation using optimal interpolation with a quasi-geostrophic model. Journal of Geophysical Research, 96, 15093-15103.

Robert, C.P. and G. Casella, 1999: Monte Carlo Statistical Methods. Springer: New York.

Royle, J.A., Berliner, L. M., Wikle, C. K. and Milliff, R., 1999: A hierarchical spatial model for constructing wind fields from scatterometer data in the Labrador 
Sea. Case Studies in Bayesian Statistics, C. Gatsonis et al. (eds.), SpringerVerlag, 367-382.

Thiebaux, H.J., 1975: Experiments with correlation representations for objective analysis. Monthly Weather Review, 103, 617-627.

Wikle, C.K., Berliner, L.M., and N. Cressie, 1998: Hierarchical Bayesian spacetime models. Journal of Environmental and Ecological Statistics, 5, 117-154.

Wikle, C.K., R.F. Milliff, D. Nychka, and L.M. Berliner, 2001: Spatiotemporal hierarchical Bayesian modeling: Tropical ocean surface winds. Journal of the American Statistical Association, 96, 382-397. 
Table 1: Modeling Scenarios

\begin{tabular}{rll} 
Scenario & $\boldsymbol{\mu}_{B}$ & $\sigma_{B}^{2}$ \\
\hline Case I: & Spatially varying; e.g., Figure 2e, 3e & $1.1 \times 10^{12} \mathrm{~m}^{4} \mathrm{~s}^{-2}$ \\
Case II: & Same as Case I & $1.0 \mathrm{~m}^{4} \mathrm{~s}^{-2}$ \\
Case III: & Constant, $\mu_{B}=-3.5 \times 10^{5} \mathrm{~m}^{2} \mathrm{~s}^{-1}$ & $1.1 \times 10^{12} \mathrm{~m}^{4} \mathrm{~s}^{-2}$ \\
\hline
\end{tabular}




\section{FIGURE CAPTIONS}

Figure 1. NSCAT data and study domain area.

Figure 2. Streamfunction posterior mean, standard deviation, and realizations for Case I, with $\boldsymbol{\mu}_{B}$ as shown in panel (e) by the solid line (the prior and posterior mean are equivalent for the model presented here) and $\sigma_{B}^{2}=1.1 \times 10^{12} \mathrm{~m}^{4} \mathrm{~s}^{-2}$; contour values should be multiplied by $10^{6} \mathrm{~m}^{2} \mathrm{~s}^{-1}$.

Figure 3. Streamfunction posterior mean, standard deviation, and realizations for Case II with $\boldsymbol{\mu}_{B}$ spatially varying as shown by the solid line in panel (e) and $\sigma_{B}^{2}=1.1 \mathrm{~m}^{4} \mathrm{~s}^{-2}$; contour values should be multiplied by $10^{6} \mathrm{~m}^{2} \mathrm{~s}^{-1}$. Note the boundary realizations are collinear because of the extremely small prior variance on the boundary process.

Figure 4. Streamfunction posterior mean, standard deviation, and realizations for Case III with $\mu_{B}=-3.5 \times 10^{5} \mathrm{~m}^{2} \mathrm{~s}^{-1}$ for all boundary locations and $\sigma_{B}^{2}=$ $1.1 \times 10^{12} \mathrm{~m}^{4} \mathrm{~s}^{-2}$; contour values should be multiplied by $10^{6} \mathrm{~m}^{2} \mathrm{~s}^{-1}$. 


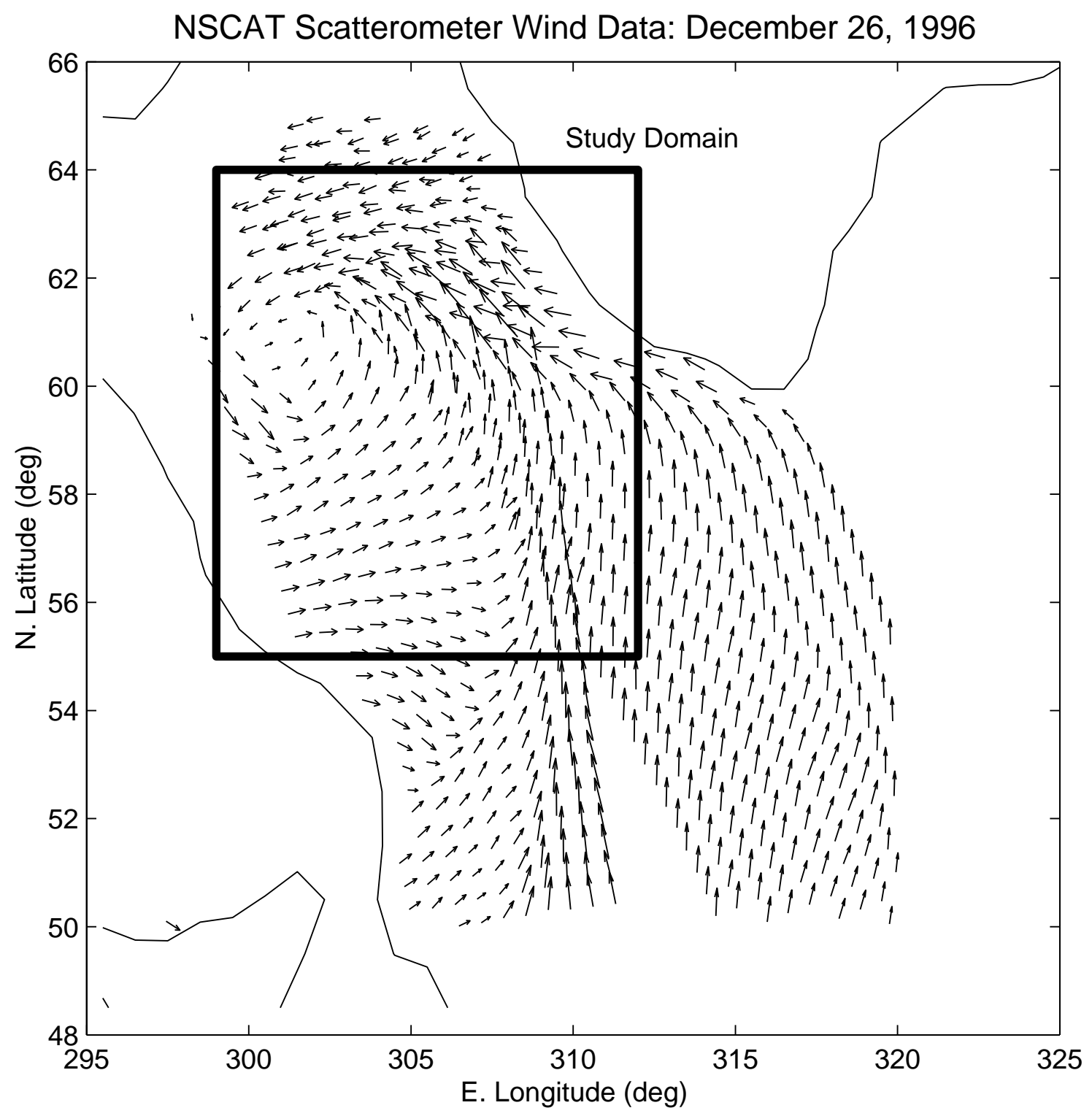

Figure 1: NSCAT data and study domain area. 

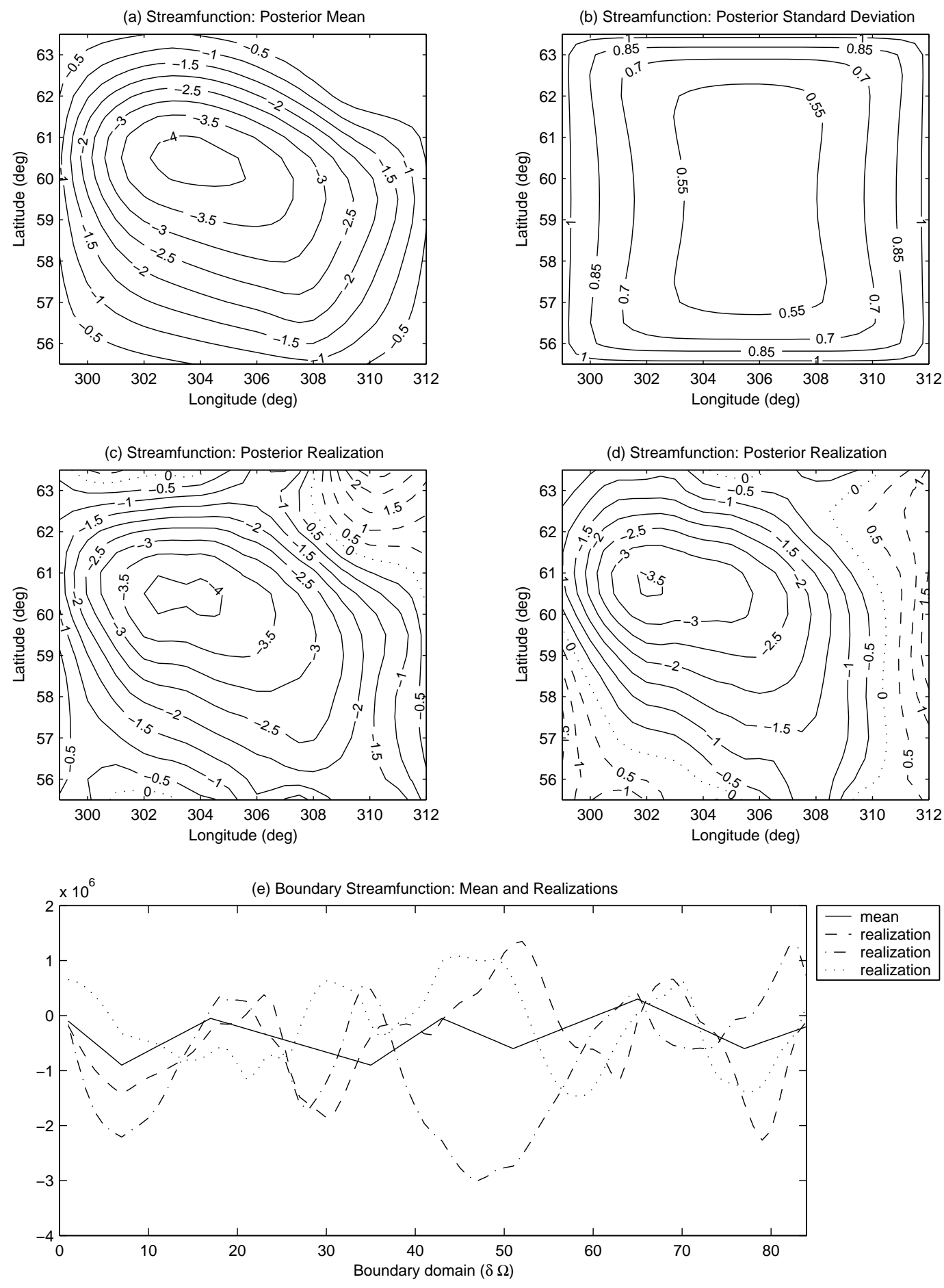

Figure 2: Streamfunction posterior mean, standard deviation, and realizations for Case I, with $\boldsymbol{\mu}_{B}$ as shown in panel (e) by the solid line (the prior and posterior mean are equivalent for the model presented here) and $\sigma_{B}^{2}=1.1 \times 10^{12} \mathrm{~m}^{4} \mathrm{~s}^{-2}$; contour values should be multiplied by $10^{6} \mathrm{~m}^{2} \mathrm{~s}^{-1}$. 

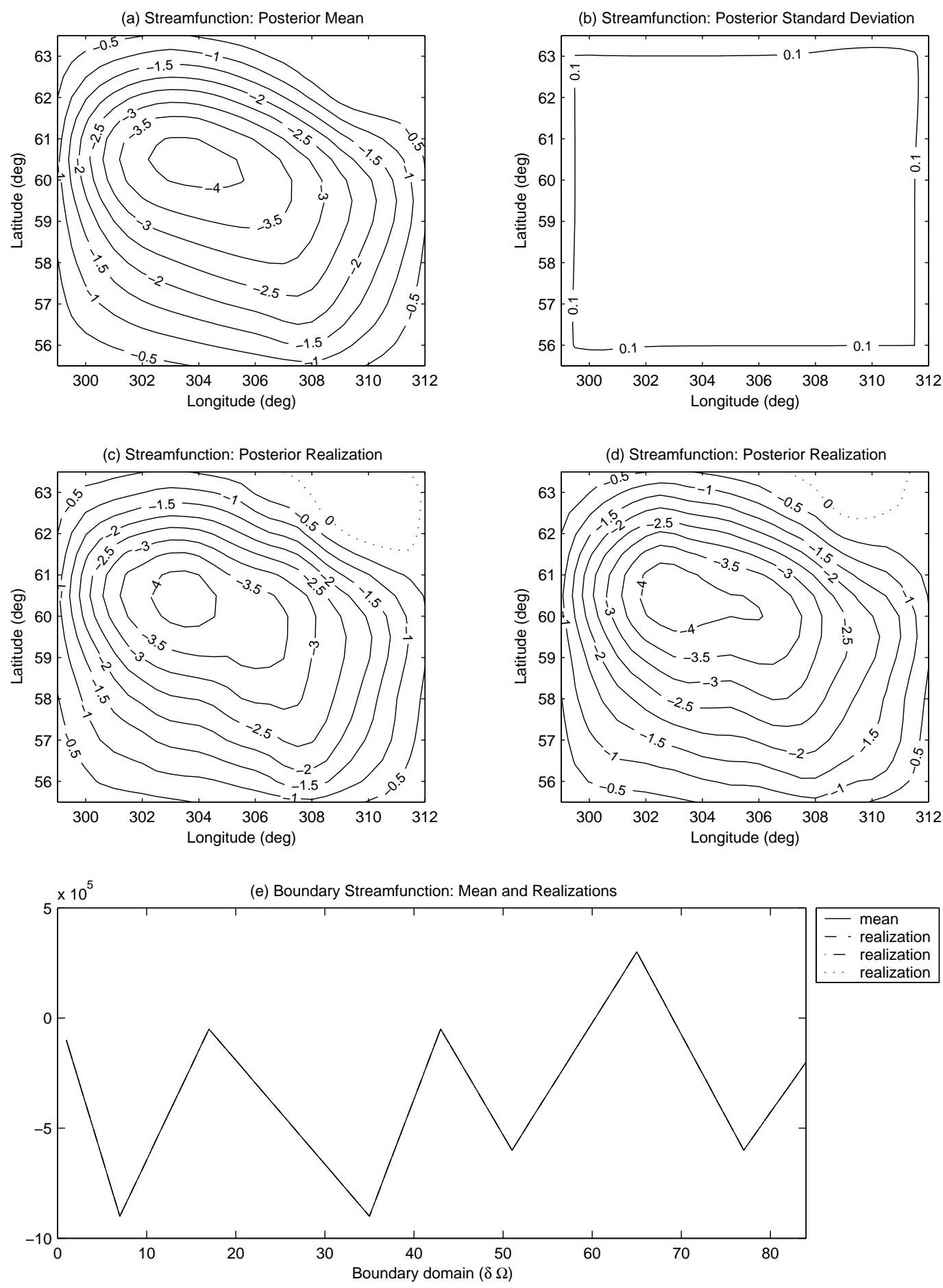

Figure 3: Streamfunction posterior mean, standard deviation, and realizations for Case II with $\boldsymbol{\mu}_{B}$ spatially varying as shown by the solid line in panel (e) and $\sigma_{B}^{2}=1.1 \mathrm{~m}^{4} \mathrm{~s}^{-2}$; contour values should be multiplied by $10^{6} \mathrm{~m}^{2} \mathrm{~s}^{-1}$. Note the boundary realizations are collinear because of the extremely small prior variance on the boundary process. 

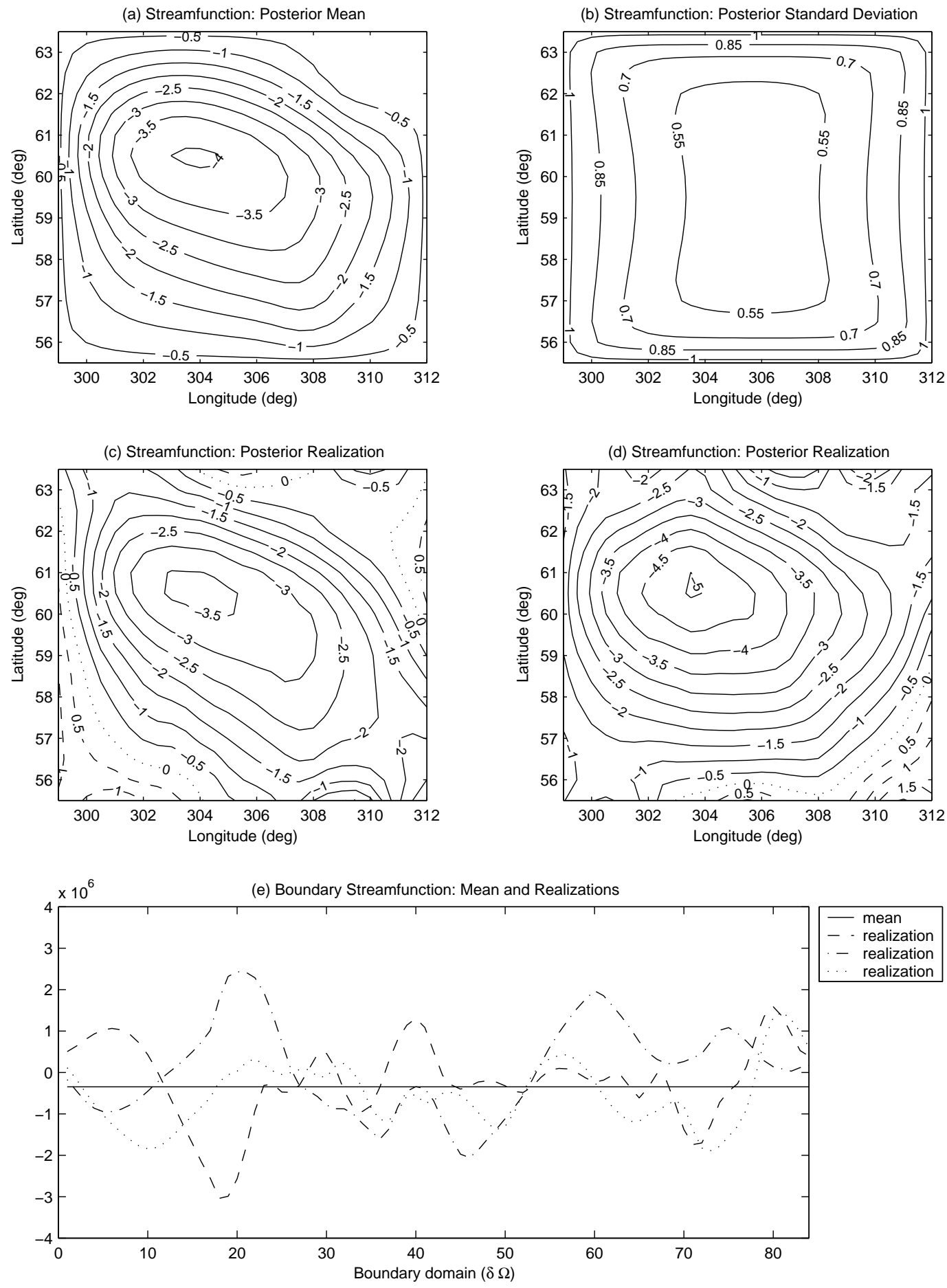

Figure 4: Streamfunction posterior mean, standard deviation, and realizations for Case III with $\mu_{B}=-3.5 \times 10^{5} \mathrm{~m}^{2} \mathrm{~s}^{-1}$ for all boundary locations and $\sigma_{B}^{2}=$ $1.1 \times 10^{12} \mathrm{~m}^{4} \mathrm{~s}^{-2}$; contour values should be multiplied by $10^{6} \mathrm{~m}^{2} \mathrm{~s}^{-1}$. 